\title{
Hypercoagulation and elevation of blood triglycerides are characteristics of Kawasaki disease
}

\author{
Xi Chen ${ }^{1,2}$, Zhen-Wen Zhao ${ }^{3}$, Lin Li ${ }^{3}$, Xue-Jun Chen ${ }^{1,2}$, Hui Xu1, Jin-Tu Lou ${ }^{1,2}$, Lin-Jie Li ${ }^{1}$, Li-Zhong Du ${ }^{1,2}$ \\ and Chun-Hong Xie ${ }^{1 *}$
}

\begin{abstract}
Background: Cardiovascular damages poses risks to children with Kawasaki disease (KD). Although hypertriglyceridemia and hypercholesteremia are risk factors of cardiovascular damages, studies on the blood lipid metabolism in KD are still limited. This study aims to analyze the blood lipids and coagulation in KD.

Methods: Triglyceride (TG) and cholesterol levels in the plasma and serum from 20 children with KD were examined in comparison with 10 healthy children (HC) as well as 10 children with high fever from identified bacterial infections (BT). Using electrospray ionization mass spectrometry, we profiled the lipid species. Blood coagulation was analyzed. Statistics was analyzed by one-way ANOVA using SigmaStat.

Results: We found that in KD, plasma TG level was significantly increased, but not serum TG. A total of 19 molecular species of TG were identified, and they were all increased in KD and BT patients, and more pronounced in KD. On the other hand, major molecular species of plasma phosphotidylcholine and lyso-phosphotidylcholine were decreased in KD and BT. Pronounced hypercoagulation was found in KD blood.

Conclusion: Our data indicate hyperlipidemia in KD, especially for TG, which contributes to the hypercoagulation and the potential risk of cardiovascular damages. Evaluation of blood lipid levels in severe KD patients could provide valuable information for treatment and prognosis, thus would be worthy of consideration.
\end{abstract}

Keywords: Kawasaki disease, Lipidomics, Triglyceride, Plasma, Serum, Hypercoagulation, Thromboelastography

\section{Background}

First described by T. Kawasaki [1], Kawasaki disease (KD) is a systematic syndrome with unknown etiology. It broadly affects blood vessels, skin, mucous and lymph nodes, and is typically manifested by fever and systemic vasculitis, and poses risk to patients' life when the coronary arteries are affected. KD is most common in children under 5 years of age, males and of Asian ethnicity. It is attributed to immune disease since $\mathrm{KD}$ vaculitis is accompanied by increase of inflammatory cells and cytokines without identified pathogens, and is responsive to intravenous immunoglobulin and corticosteroid therapy.

\footnotetext{
* Correspondence: xch_hz@hotmail.com

'Department of Cardiology, Children's Hospital, Zhejiang University School of Medicine, No.3333 Bin-Sheng Road, Bin-Jiang Dist, Hangzhou, Zhejiang 310052, China

Full list of author information is available at the end of the article
}

In our provincial children's hospital in Zhejiang, China, about $350 \mathrm{KD}$ patients are admitted each year and 5-6\% are accompanied by coronary artery lesions, while severe cardiovascular damages including and valvular insufficiencies and cardiogenic shock are rare complications [2]. Intravenous immunoglobulin is our first line therapy with the responsive rate of $85 \%$, yet the risk to develop cardiovascular damages is increased in intravenous immunoglobulin-resistant patients. The recovered KD patients have a higher risk to develop atherosclerosis in adult years [3-6].

Although the accompanying and secondary cardiovascular damages pose great risk to $\mathrm{KD}$ children, studies on the blood lipid metabolism in KD are still limited. Biolipids are not only compositional materials as compartmental barrier, but also signaling molecules that mediates cellular functions. Lipid metabolites are currently used as biomarkers 
for the diagnosis and prognosis of cardiovascular diseases, cancer, and neurological diseases. In recent years, tandem mass spectrometric technique has greatly facilitated the quantitative and qualitative studies of biolipids, and lipidomics analysis is currently being expanded as a diagnostic tool for the benefit of personalized medicine [7]. The blood contains thousands of biolipid species, the levels are determined by both food intake and acquired or secondary factors including individual genetics and metabolic traits. In this study, we analyzed the blood lipid profile in $\mathrm{KD}$ children in comparison with healthy children (HC) and children with high fever $\left(>38^{\circ} \mathrm{C}\right)$ from diagnosed bacterial infections (BT) from the same age group by biochemical methods as well as electrospray ionization mass spectrometry (ESI MS).

Blood triglyceride (TG) is distributed between chylomicrons and very-low density lipoprotein. It has been recognized that hypertriglyceridemia is a risk factor of cardiovascular damages independent of cholesterol [8-12]. Our study has analyzed the specific change of TG molecular species as it is the major component of blood lipids. There is a potential link established between increased blood TG and low-grade inflammation $[10,12]$ that is also manifested in KD patients.

\section{Results}

\section{Hypercoagulation in KD patients}

KD patients were diagnosed based on symptoms $[1,13]$. On the day when fasting blood samples were collected, the patients were still feverish, with $\mathrm{C}$-reaction protein (CRP) and erythrocyte sedimentation rate (ESR) significantly exceeding the normal range (Table 1), suggesting persisted inflammation.

From Fig. 1a, the platelet number was $349 \pm 169 \times 10^{\wedge} 9 / \mathrm{L}$ in the KD group, and $276 \pm 39 \times 10^{\wedge} 9 / \mathrm{L}$ in the group of healthy controls (HC). The fibrinogen level was $4.88 \pm 0.71 \mathrm{~g} / \mathrm{L}$ in the KD group, and was $2.04 \pm 0.48 \mathrm{~g} / \mathrm{L}$ in the $\mathrm{HC}$ group. The level of $\mathrm{D}$-dimer was $1.55 \pm$ $1.74 \mathrm{mg} / \mathrm{L}$ in the KD group, and was $0.48 \pm 0.52 \mathrm{mg} / \mathrm{L}$ in the $\mathrm{HC}$ group. The statistical differences were significant $(P<0.05)$. Prothrombin time $(\mathrm{PT})$, activated partial thromboplastin time (APTT) and thrombin time (TT) were all within the normal range and showed no significant differences between all sample groups (Additional file 1: Table S1).

Thromroelastography (TEG) monitors the process of blood clot formation and retraction dynamically. It was first established by Harter in 1948, and has been proved valuable in examining coagulation specifically [14, 15]. Our facility produces the following parameters of significance: $\mathrm{R}$ (reaction time) stands for the time for initial fibrin formation, and prolongation of $\mathrm{R}$ value is due to coagulation factor deficiencies; $\mathrm{K}$ (coagulation time) measures the speed of clot strengthening, and increased $\mathrm{K}$
Table 1 Summarized clinical information of the 20 patients with Kawasaki disease recruited in our study on the day of blood sample collection

\begin{tabular}{llllcll}
\hline & Age & Sex & Days of fever & Body temp, ${ }^{\circ} \mathrm{C}$ & $\mathrm{CRP}^{\mathrm{a}}$ & $\mathrm{ESR}^{\mathrm{b}}$ \\
\hline 1 & $4 \mathrm{y} 9 \mathrm{~m}$ & $\mathrm{M}$ & 3 & 38.8 & 83 & 66 \\
2 & $5 \mathrm{y} 1 \mathrm{~m}$ & $\mathrm{~F}$ & 5 & 38.5 & 94 & 64 \\
3 & $4 \mathrm{~m} 23 \mathrm{~d}$ & $\mathrm{M}$ & 5 & 36.8 & 110 & 62 \\
4 & $5 \mathrm{y} 6 \mathrm{~m}$ & $\mathrm{~F}$ & 7 & 36.8 & $>160$ & 75 \\
5 & $8 \mathrm{~m} 13 \mathrm{~d}$ & $\mathrm{M}$ & 8 & 39.0 & 23 & 76 \\
6 & $3 \mathrm{y} 10 \mathrm{~m}$ & $\mathrm{~F}$ & 5 & 38.5 & 128 & 93 \\
7 & $2 \mathrm{y} 9 \mathrm{~m}$ & $\mathrm{M}$ & 5 & 39.5 & 63 & 66 \\
8 & $1 \mathrm{y} 1 \mathrm{~m}$ & $\mathrm{M}$ & 5 & 38.0 & 139 & 66 \\
9 & $3 \mathrm{y} 11 \mathrm{~m}$ & $\mathrm{~F}$ & 5 & 39.1 & 98 & 88 \\
10 & $5 \mathrm{y} 2 \mathrm{~m}$ & $\mathrm{M}$ & 5 & 38.5 & 41 & 55 \\
11 & $1 \mathrm{y} 8 \mathrm{~m}$ & $\mathrm{M}$ & 6 & 39.5 & 69 & 55 \\
12 & $1 \mathrm{y} 6 \mathrm{~m}$ & $\mathrm{~F}$ & 6 & 38.9 & $>160$ & 57 \\
13 & $1 \mathrm{y} 10 \mathrm{~m}$ & $\mathrm{M}$ & 3 & 37.2 & 65 & 40 \\
14 & $1 \mathrm{y} 5 \mathrm{~m}$ & $\mathrm{M}$ & 5 & 40.5 & 20 & 35 \\
15 & $2 \mathrm{y}$ & $\mathrm{F}$ & 3 & 39.1 & $>160$ & 73 \\
16 & $5 \mathrm{y} 4 \mathrm{~m}$ & $\mathrm{M}$ & 4 & 38.8 & 95 & 75 \\
17 & $6 \mathrm{~m} 17 \mathrm{~d}$ & $\mathrm{M}$ & 3 & 39.0 & 25 & 83 \\
18 & $1 \mathrm{y} 5 \mathrm{~m}$ & $\mathrm{M}$ & 4 & 38.5 & 40 & 62 \\
19 & $1 \mathrm{y} 2 \mathrm{~m}$ & $\mathrm{~F}$ & 3 & 38.8 & 45 & 87 \\
20 & $3 \mathrm{~m} 22 \mathrm{~d}$ & $\mathrm{M}$ & 4 & 39.0 & 29 & 66 \\
\hline
\end{tabular}

${ }^{\mathrm{a}} \mathrm{C}$-reaction protein (CRP), normal range $0-8 \mathrm{mg} / \mathrm{L}$

berythrocyte sedimentation rate (ESR), normal range $0-20 \mathrm{~mm} / \mathrm{h}$

value suggests hypofibrogenemia or platelet dysfunction; the maximum amplitude (MA) represents the clot strength, and is a parameter to judge platelet function; the coagulation index $(\mathrm{CI})$ is a parameter for the overall coagulation state. We examined $20 \mathrm{KD}$ blood samples by TEG, and the results have revealed marked hypercoagulation. Typical thromboelastographs from a healthy individual and that from a KD patient are shown (Fig. 1b, c). As summarized in Table 2, in the KD patients, the $\mathrm{R}$ value was significantly lowered ( $4.3 \pm 1.8$ compared with $5-10)$, the $\mathrm{K}$ value was within the normal range, MA was significantly increased (75.9 \pm 4.6 compared with 50-70), and so was CI $(3.9 \pm 1.8$ compared with $-3-+3)$. As judged by $\mathrm{CI}$, the occurrence rate of hypercoagulation in $\mathrm{KD}$ was $80 \%$. Most of the $\mathrm{KD}$ patients had abnormal MA (95\%) and R (80\%) values, while only $15 \%$ had abnormal $\mathrm{K}$.

\section{Increased plasma TG, but not serum TG in KD}

Both plasma and serum are being used for blood lipid testing. From the literature, the plasma is considered to be more optimal to reflect the original physiological properties compared with the serum [16]. TG and cholesterol levels from serum and plasma were both examined. As shown in Fig. 2, total plasma TG was significantly increased 


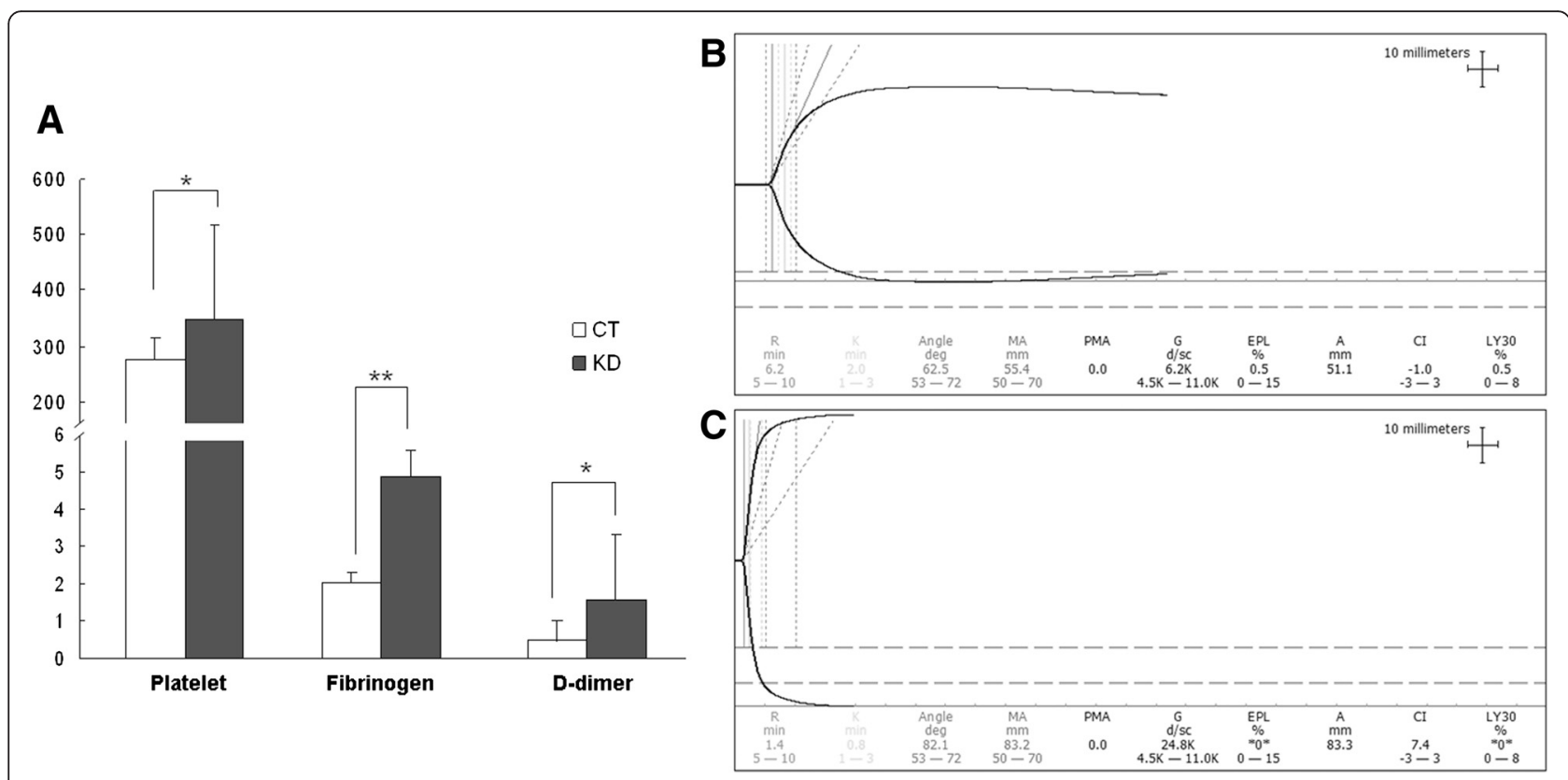

Fig. 1 Blood coagulation and thromboelastography analysis. a Comparison of platelet number, plasma fibrinogen (g/L) and D-dimer (mg/L) levels from healthy children $(\mathrm{HC})$ and Kawasaki disease $(\mathrm{KD})$. $^{*}: P<0.05$. ${ }^{*}: P<0.01$. b-c Representative thromboelastographs from blood samples with normal coagulation (b) and with hypercoagulative state in a patient with Kawasaki disease (c)

in $\mathrm{KD}$ compared with the group of $\mathrm{HC}$ and the feverish children with bacterial infections (BT). Levels of plasma TG in BT and HC groups were not statistically significant. However, examination of the serum revealed a different scenario. The TG levels in the HC and KD groups were not statistically significant, while it was significantly decreased in BT patients. Cholesterol levels were similar in all 3 groups, both in the plasma and the serum.

\section{Plasma TG profiling by mass spectrometry}

Our established high performance liquid chromatographyelectrospray ionization- quadrupole time of flight mass spectrometry (HPLC-ESI-QTOF MS)-based protocol scans over 20,000 molecular species of polar lipids. All lipids were eluted from the HSS T3 column within 12 min of elution (Fig. 3a), lyso-phosphatidylcholine (LPC) species were eluted at the first $2 \mathrm{~min}$, phosphatidylcholine (PC) species were eluted from 2 to $6 \mathrm{~min}$, while TGs were eluted from 6 to $10 \mathrm{~min}$. By performing partial least square-discriminant analysis, the 3 experimental groups were well separated (Fig. 3b) and lipid species contributing to the discriminative

Table 2 Collected parameters by thromboelastography analysis of blood samples from 20 patients with Kawasaki disease

\begin{tabular}{lllll}
\hline & $\mathrm{R}$ & $\mathrm{K}$ & $\mathrm{MA}$ & $\mathrm{Cl}$ \\
\hline Mean & 4.3 & 1.2 & 75.9 & 3.9 \\
SD & 1.8 & 0.3 & 4.6 & 1.8 \\
Reference range & $5-10$ & $1-3$ & $50-70$ & $-3-3$ \\
Abnormal rate & $80.0 \%$ & $15.0 \%$ & $95.0 \%$ & $80.0 \%$ \\
\hline
\end{tabular}

separation were picked and confirmed by LIPID MAPS. Besides the neutral lipid cholesterol, the major plasma lipid is TG and PC. Consistently, we found these two classes were the major contributing species to discriminate the separation between the 3 groups.

From the most abundant 100 molecular species of plasma polar lipids, 19 was identified as TGs, which were all significantly increased in $\mathrm{KD}$ as well as BT patients (Fig. 4 and Additional file 1: Table S2). From the 19 TGs, 5 species were significantly increased in KD compared with the BT group: TG 48:1, TG 52:1, TG, $52: 3$, TG 52:4 and TG 56:8. These are the relatively more abundant TGs, consisting $41 \%$ of total TG in KD, and $36-37 \%$ in $\mathrm{HC}$ and BT groups.

\section{Decrease of plasma PC and LPC in KD patients}

$\mathrm{PC}$ is the second abundant group of polar plasma lipids. In our study, we have observed significant changes in several PC molecular species from KD and BT groups compared with controls, including 7 PCs and 2 LPCs. Contrary from TG, PC and LPC species were decreased in patients (Fig. 5 and Additional file 1: Table S3). Of the 7 PCs of interest, 2 molecular species with relatively shorter free fatty acid (FFA) chains, PC 14:0/18:1 and PC 14:0/20:2, were more significantly decreased in $K D$, while the other 5 were more significantly decreased in BT. However, change of PC profile was not significant between KD and BT groups. Change of the two LPCs was more striking than $\mathrm{PC}$, significantly decreased in $\mathrm{KD}$ and even further decreased in BT (Fig. 5 and Additional file 1: Table S3). It 


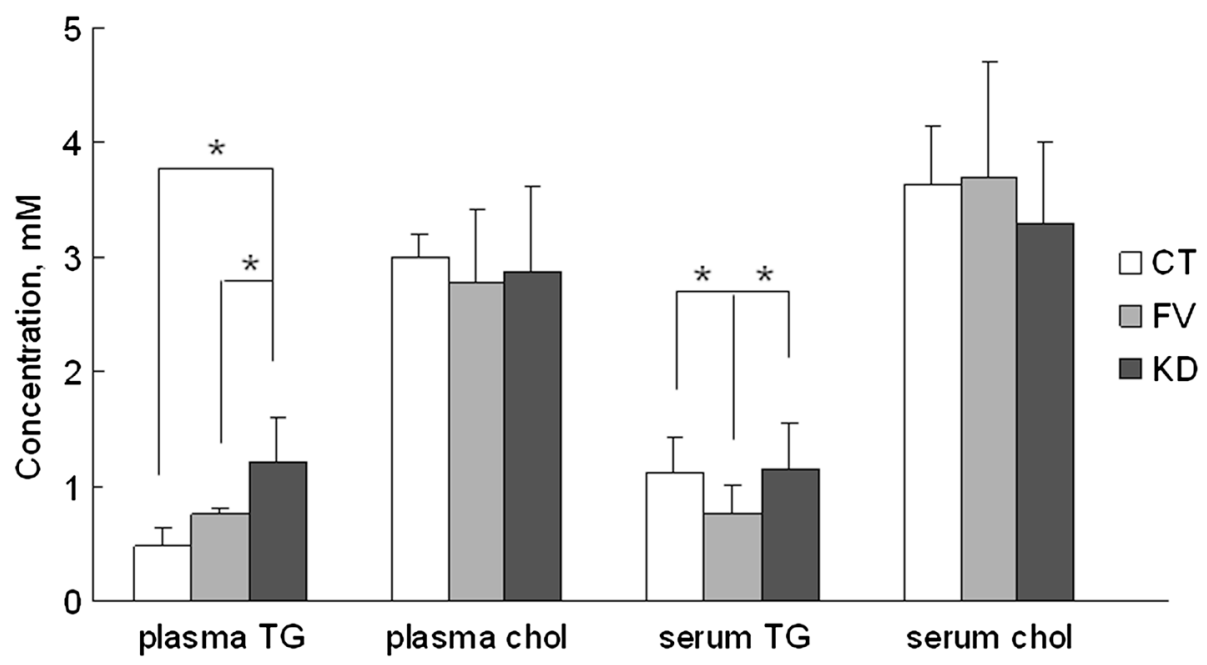

Fig. 2 Serum and plasma triglyceride and cholesterol levels. Blood biochemistry analysis of plasma and serum triglyceride (TG) and cholesterol (chol) levels from 3 groups: healthy children (CL), patients from diagnosed bacterial infections (BT) and patients with Kawasaki disease (KD). *P: $<0.05$

was possible to separate $\mathrm{KD}$ and $\mathrm{BT}$ by the $\mathrm{LPC}$ content because of the extremely sharp decrease of LPC in BT patients.

\section{Discussion}

\section{Hypercoagulation in KD and anti-coagulation therapy}

Platelet counting and coagulation analysis revealed significant increase of plate number and levels of fibrinogen and D-dimer in KD (Fig. 1a), suggesting hypercoagulation as a common complication of KD patients. As a point-ofcare testing method, TEG monitors the dynamic formation of blood clots, and is advantageous than traditional measurement of coagulation factors in its fast testing speed and the ability to further discriminate between platelet- and fibrinogen-associated coagulation. Although no increase of prothrombin time, activated partial thromboplastin time and thrombin time was detected, TEG indicates that blood coagulation was faster in $80 \% \mathrm{KD}$ patients (Table 2). Change in MA that represents platelet dysfunction was manifested in $95 \%$ of $\mathrm{KD}$ patients, while the $\mathrm{K}$ value associated with fibrinogen was mostly within the normal range. Based on our data and previous clinical experience, increased fibrinogen level does not necessarily lead to significant fastened blood coagulation. Fibrinogen is an acute-phase protein produced in the liver upon stimulation of a broad spectrum of stress signals including
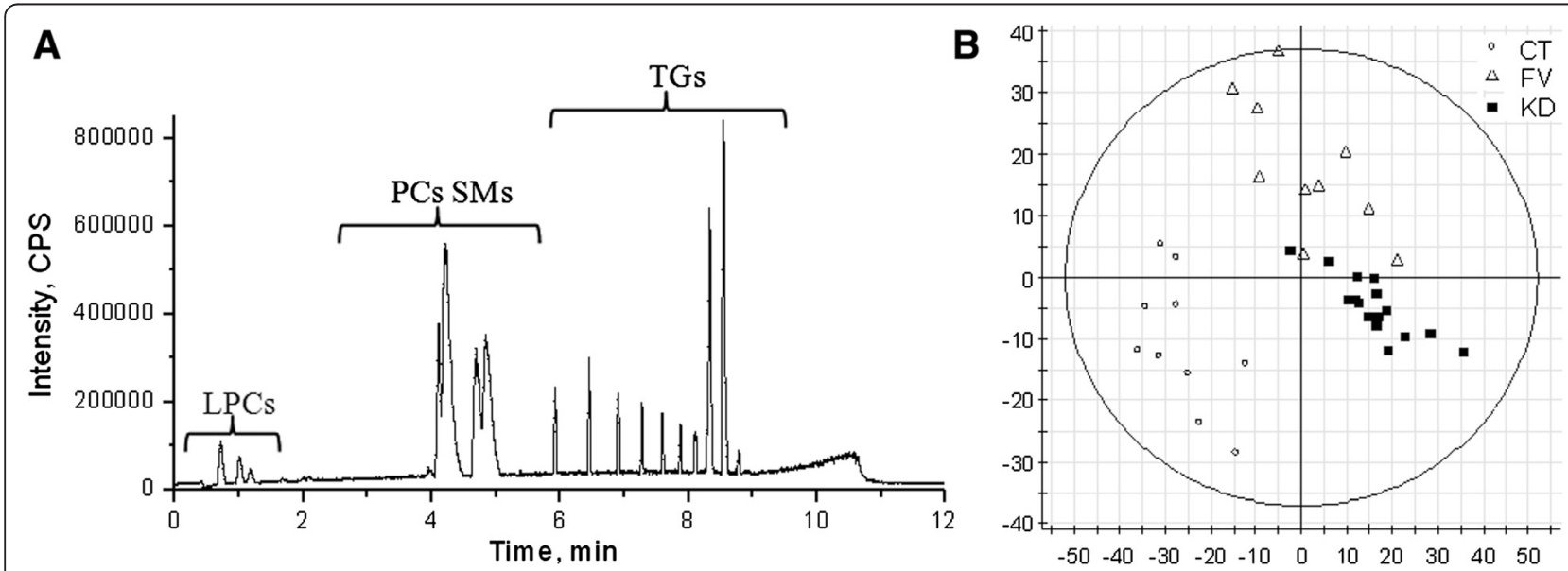

Fig. 3 Detection of polar lipids by UPLC-ESI-MS and discriminant analysis between groups. a Total ion current of representative plasma lipid extract from UPLC-ESI-QTOF MS. Within the 12 min of elution time of each sample, lyso-phosphatidylcholine (LPC), phosphatidylcholine (PC) and sphingomyelin (SM) were eluted serially, followed by triglyceride (TG). $\mathbf{b}$ The PLS-DA scoring chart by EZ info software of plasma lipids detected by the positive ESI mode MS from three groups (HC: control; BT: feverish with bacterial infections; KD: Kawasaki disease) indicates separation of sample groups 


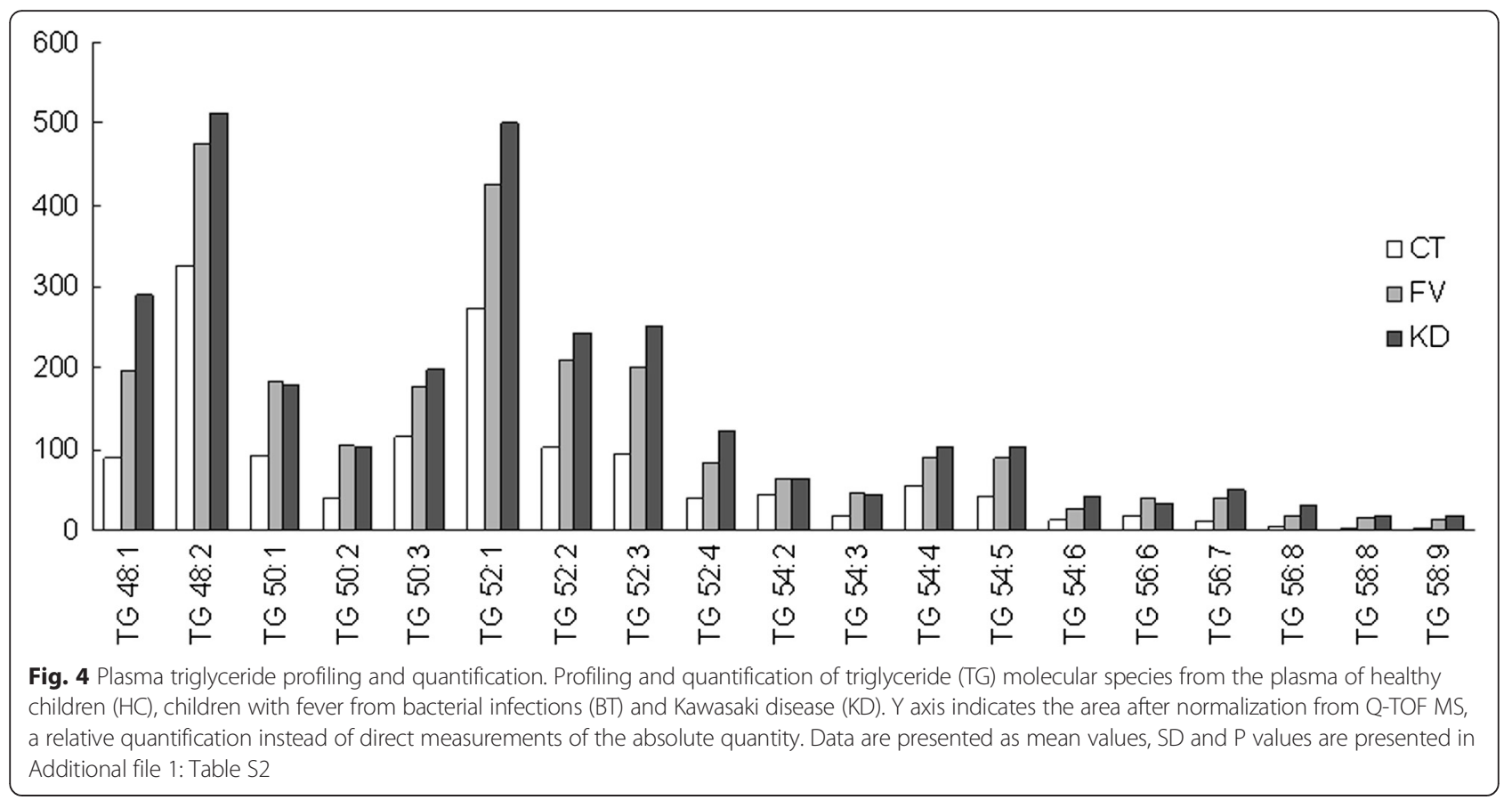

inflammation, trauma, etc. Compared with the fibrinogen level, activation of platelet is a more significant contributing factor of fastened blood coagulation. Both PT/APTT and TEG analysis suggested that hypercoagulation in KD patients was mainly attributed to platelet over-activation, although hyperfibrinogenemia was also involved.
Previous retrospective research identified secondary thrombocytopenia as a complication in some KD patients $[17,18]$, and is a risk factor for severe cardiovascular damages including coronary artery aneurysm and acute myocardial infarction $[19,20]$. These studies also suggested that thrombocytopenia in KD was a result of intravascular

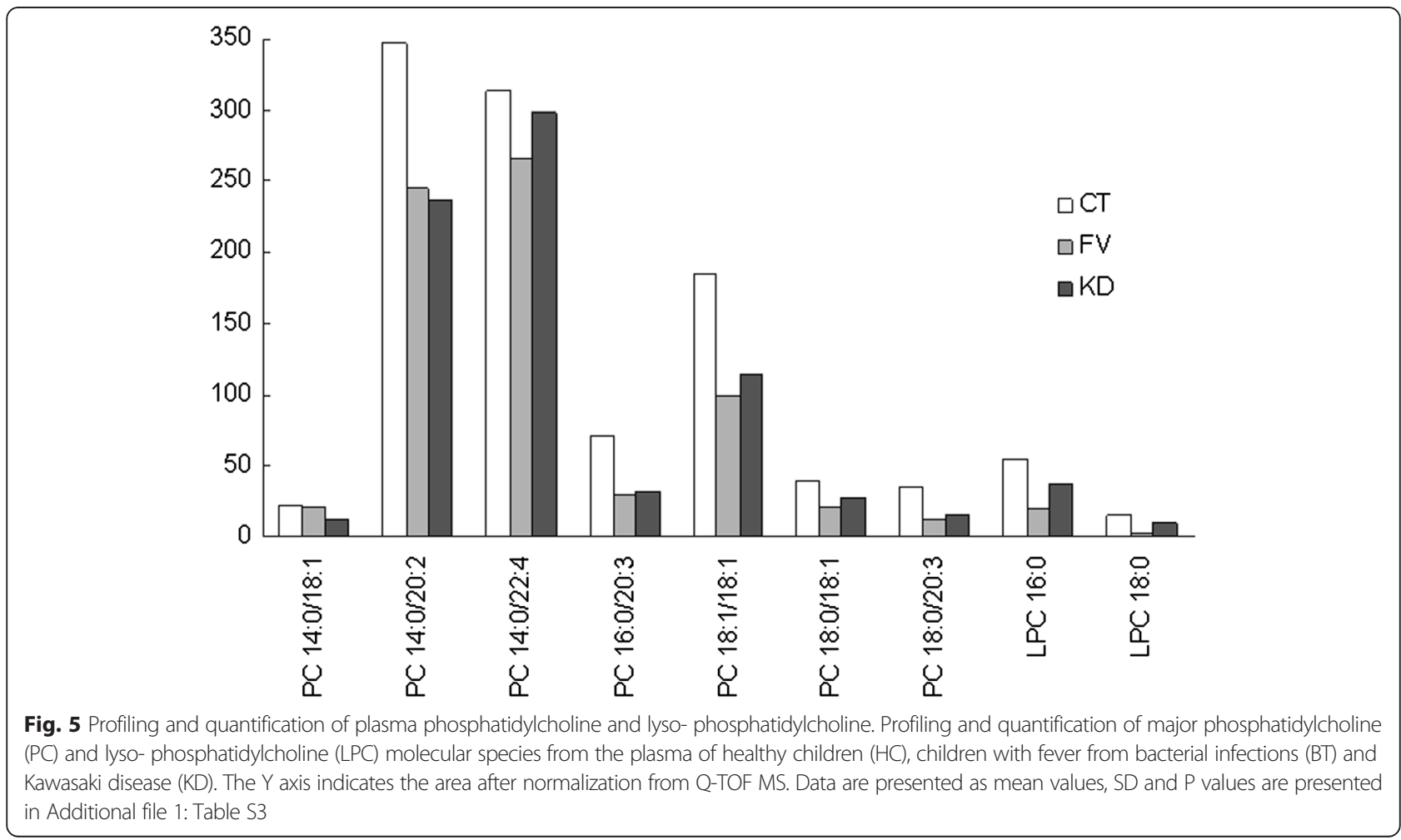


coagulation [20]. Furthermore, although not occurred in our hospital, literature reported a couple of KD cases with a rare but highly dangerous complication: disseminated intravascular coagulation (DIC), the dysregulated balance between coagulation and fibrinolysis [21, 22]. DIC could occur in KD patients without evidence of pathogen invasion. Based on our findings and the literature, we advise the clinicians to be cautious with the hypercoagulative state in KD patients, especially for the severe and intravenous immunoglobulin-resistant cases to prevent serious complications. Guided anti-coagulation therapy could be of benefit to patients with positive laboratory findings and other risk factors.

\section{Abnormal plasma TG in KD children}

We have examined the levels of TG and cholesterol from both serum and plasma, and found plasma TG levels were significantly increased in KD compared with $\mathrm{HC}$ and BT groups, while there was no difference in the cholesterol levels (Fig. 2). TG is the major component of very-low density lipoprotein and chylomicrons, while cholesterol is mainly transported in low density lipoprotein. Cholesterol and low density lipoprotein are long considered as a risk factor for cardiovascular damages, but the pathological role of TG and very-low density lipoprotein in cardiovascular damages has only been revealed in recent years. TG hydrolysis in arterial wall foam cells could lead to development of atherosclerosis plaques [11]. Lipolysis of TG-rich lipoproteins produces potentially toxic oxidized FFAs, monoacylglycerols and other molecules to activate immune cells and stimulate the production of cytokines along the artery wall [23, 24]. Genome-wide association studies also suggested causal association between elevated TG and cardiovascular damages $[25,26]$. Thus increased blood TG levels could exacerbate inflammation and arterial damage. Our data suggested that plasma TG metabolism is a more sensitive parameter than cholesterol in the sterile inflammation of KD compared with bacteria-induced infection. Such lipid metabolic change partly explains why KD patients have extremely high risks to develop cardiovascular damages such as arteriosclerosis. Consistently, in recent publications, increase of serum TG (plasma not examined) in late stage $\mathrm{KD}$ was discovered [27], and use of statins was shown to benefit KD patients to reduce the inflammation and the endothelial function [28]. In current clinical practice, both plasma and serum are used to perform blood lipid analysis. Although there is still no general guidance in discriminating the two categories of samples in blood lipid testing, our data suggest that blood coagulation may affect the lipid profile, such as TG, thus plasma could be a more reliable object as other researchers have reported [16].

During our study, we found that the TG levels were clinically reported normal in all patients, however, our comparative analysis revealed statistic difference between $\mathrm{HC}, \mathrm{KD}$ and BT children. This is watchful that the current blood lipid reference range in pediatric laboratory medicine may not be suitable, at least not for the Chinese children under 5 years of age targeted by our research. In detail, our currently applied upper value for plasma cholesterol is $4.4 \mathrm{mM}$, and is $1.5 \mathrm{mM}$ for TG, lower than the standards applied for adults $(5.2 \mathrm{mM}$ for cholesterol and $1.7 \mathrm{mM}$ for TG). Our clinical reference range (plasma cholesterol $<=4.4 \mathrm{mM}$, plasma $\mathrm{TG}<=1.5 \mathrm{mM}$ ) is slightly lower than that applied in adults, either comparable [29] or not comparable [30,31] with data obtained from same age group of children from other countries. Considering that study on the epidemiology of hypertriglyceridemia in Chinese children is still very limited in number, we advice re-evaluation of the current standards by further larger-scale analysis to better discriminate the healthy and diseased states in blood lipid metabolism during childhood.

\section{Profiling of plasma TG molecular species in Chinese children by ESI MS lipidomics analysis}

Instead of exhaustive identification of all lipids as some researchers [32, 33], we selected the lipid species contributing to the difference between the three sample groups from the top 100 abundant species. 19 TG species were the most abundant in prevalence and the most significant contributing components to differentiate the 3 sample groups (Fig. 4). All the 19 TG species were increased in KD and BT patients, and more significantly in $\mathrm{KD}$, further supporting the increase of the absolute TG level in the diseased conditions and a general malregulation of TG metabolism. From our data, in Chinese children under 5 years of age, the most dominating TG species were TG 48:2, TG 52:1, TG 50:3, TG 52:2, TG 52:3, TG 50:1 and TG 48:1, contributing to over $70 \%$ of total TG (Additional file 1: Table S1). Palmatic acid (16:0) and oleic acid (18:1) were the most common FFA chains in identified TGs, and the average total carbon number was under 54 . This is less variable and much longer than the reported result based on the elder western populations [16, 34]. The FFA composition is determined by both diets and the metabolic mechanisms, both should contribute to the difference between our target population and others'.

In 2014, several reports were published based on a large Cohort study named San Antonio Family Heart Study covering over 1200 adult Mexican Americans. Plasma lipid composition was found to be inheritable and associated with risk of cardiovascular damages [35, 36]. In these studies, $43 \mathrm{TG}$ molecular species were identified by ESI MS, and the statistical distribution of various species was associated with age, sex and body mass index [37]. In another recent study with the intention to 
identify lipid biomarkers for cardiovascular damages [34], shotgun lipidomics detected 135 lipid species, among which TG 54:2 has been found to be a risk factor. Certain species of TG were attributed to childhood obesity [38] and insulin resistance [39]. Our analysis of molecular species of TG in the blood suggest metabolic disorder of TG in both bacteria-induced and sterile inflammation, especially the latter. Further enlarged samples may be helpful to identify whether it is a risk factors for KD-associated cardiovascular damages. Discovery of novel lipid biomarkers will facilitate diagnosis of severe complications of $\mathrm{KD}$, and to discover more therapeutic targets in the future.

Besides increase of TG, we also found marked decrease of PC and LPC in patients. Unlike TG, change of PC and LPC was more pronounced in the fever group caused by bacteria infection (Fig. 5). Our fever group is composed of children with pneumonia, and in our hospital, such cases are mostly caused by S. pneumoniae, other pathogens include haemophilus influenzae, mycoplasma pneumoniae and Klebsiella pneumoniae (intercommunications). The underlying mechanism of TG and PC/LPC malregulation remains to be discovered. In KD patients, blood cytokines levels including IL-1, IL-6, IL-10, TNF $\alpha$, and $\mathrm{BTN} \gamma$ were significantly increased $[40,41]$. There was laboratory evidence showing TNF $\alpha$, IL-1 and other cytokines could induce TG synthesis [42-44], meanwhile, association of cytokine patterns with TG levels have been observed in human $[45,46]$. Research on the regulation of inflammatory factors on lipid metabolism will be informative. Based on previous literature reports, PPAR $\gamma$ was increased in KD and could be a potential anti-inflammatory candidate target for cardiovascular damages in $\mathrm{KD}$ cases [47]. It remains to be confirmed if PPAR $\gamma$ could serve as a promising therapeutic target for KD.

In summary, our research has identified pronounced change in plasma TG, PC and LPC in KD patients with detailed profiling of molecular species. Increase of blood TG, together with hypercoagulation in KD imply the necessity to control blood lipid levels as well as anti-coagulation therapy, such as use of statins.

\section{Conclusions}

Our study demonstrated hyperlipidemia in KD children, especially for TG species, which contributes to hypercoagulation and the potential risk of cardiovascular damages, thus would be worthy of further evaluation in severe KD patients to provide information to guide treatment and prognosis. Meanwhile, our data suggested malregulation of blood lipid metabolism in KD, and should be subjected to further investigations.

\section{Methods}

\section{Patients and controls}

This study was approved by the ethics committee of the Children's Hospital, Zhejiang University School of Medicine. The study protocol conforms to the ethics guidelines of the 1975 Declaration of Helsinki, as was reflected in the a priori approval provided by the institution's human research committee. Informed consent was obtained from each patient's parent or guardian.

Fasting blood samples were collected from $20 \mathrm{KD}$ patients, 10 patients of bacterial pneumonia with body temperature above $38{ }^{\circ} \mathrm{C}$ (bacterial infection group, BT), and 10 healthy children for routine body examination (healthy control group, $\mathrm{HC}$ ). All patients were admitted to the hospital ward from June to October, 2013. The KD patients were 13 boys and 7 girls aged between 4-month to 5-year-6-month, the median age was 2year-9-month. At the time of blood collection, all KD patients were in the acute stage with fever above $37^{\circ} \mathrm{C}$ for over 3 days and without intravenous immunoglobulin administration, which was proved to be effective to all cases in the later treatments. The clinical information of KD patients is summarized in Table 1.

\section{Processing of blood samples, blood biochemistry and thromboelastography analysis}

Serum samples were subjected to laboratory test for CRP, ESR, TG/cholesterol levels and other parameters by an automatic biochemistry analyzer (DPP modular, Roche, USA). Blood samples with the presence of sodium citrate as anti-coagulant were analyzed by an automatic blood analyzer (Mindray BC-5300, China) for the platelet number, etc. Levels of fibrinogen and D-dimer were analyzed by a full-automatic coagulation analyzer (Sysmex CA1500, Japan).

Thromroelastography (TEG) was collected from a TEG5000 Haemoscope (Niles, USA), with sodium citrate as anti-coagulant and kaolin as coagulation initiator. In TEG analysis, $1 \mathrm{~mL}$ whole blood is placed in a rotating metal cuvette heated to $37^{\circ} \mathrm{C}$, as fibrin strands form between the wall of the cuvette and a piston suspended in the sample, the rotational motion gets transferred to the piston, and an electronic amplification system allows the characteristic tracing to be recorded.

Blood samples with anti-coagulant were spun at $3000 \mathrm{rpm}$ for $10 \mathrm{~min}$, supernatants (plasma) were aliquoted into siliconized eppendorf tubes (SafeSeal, PGC Scientifics, USA), frozen in dry ice and stored at $-80^{\circ} \mathrm{C}$ until use for lipid extraction and measurement of plasma TG/cholesterol.

Mass spectrometry analysis of lipids extracted from plasma HPLC-grade acetonitrile, isopropanol, formic acid and ammonium formate were purchased from Sigma or Fisher Scientific (USA). Ultrapure water from Milli-Q purification system (Millipore, USA) was used. 
Plasma lipids were extracted by isopropanol [48]. In brief, $10 \mu \mathrm{L}$ of plasma was mixed with $500 \mu \mathrm{L}$ of isopropanol, after vortexing and centrifugation $(10,000 \times \mathrm{g}$, $5 \mathrm{~min}$, room temperature), the supernatant was retained and $2 \mu \mathrm{L}$ of which was loaded into mass spectrometer with an auto sampler through a LC system (UPLC Hclass, Waters, USA). A HSS T3 column $(1.8 \mu \mathrm{m}, 2.1 \mathrm{~mm}$ ID $\times 100 \mathrm{~mm}$, Waters, USA) maintained at $65{ }^{\circ} \mathrm{C}$ was used for separation of lipids. The mobile phase A was isopropanol/acetonitrile/formic acid (90:10:0.1, v/v/v) containing $10 \mathrm{mM}$ ammonium formate; the mobile phase $\mathrm{B}$ was acetonitrile/water/formic acid (60:40:0.1, v/v/v) containing $10 \mathrm{mM}$ ammonium formate. The UPLC separations were $12 \mathrm{~min} /$ sample using the following scheme: 1 ) 0 min, $60 \%$ B; 2) 10 min, $1 \%$ B; 3) 12 min, $60 \%$ B. All changes were linear with a flow rate of $400 \mu \mathrm{L} / \mathrm{min}$.

Lipid profile analysis was performed using ESI Q-TOF MS (Xevo G2, Waters, USA). Both the nebulizer and desolvation gases were nitrogen. Typical operating condition was set as follows: capillary voltage $3.0 \mathrm{kV}$, sampling cone voltage $40 \mathrm{~V}$, desolvation gas temperature $500{ }^{\circ} \mathrm{C}$, source temperature $150{ }^{\circ} \mathrm{C}$, nebulization gas flow $40 \mathrm{~L} / \mathrm{h}$, and desolvation gas flow $400 \mathrm{~L} / \mathrm{h}$. The $\mathrm{m} / \mathrm{z}$ range detected was from 50 to 1200 . The positive ion $\mathrm{MS}^{\mathrm{E}}$ mode was used for data acquisition.

\section{Statistical analysis}

Data obtained by MS were processed by Markerlynx XS and EZ info softwares (Waters, USA). Markerlynx XS was used to carry out peak discrimination, filtering, and retention time alignment, yielding $>20000$ peaks between the retention time of $0-12 \mathrm{~min}$. To investigate global lipids alterations, all observations acquired were integrated and co-analyzed using partial least square-discriminant analysis by EZ info software. The VIP-plot in EZ info software was further carried out to select variables as potential markers for distinguishing patients from controls. The VIP value reflects the influence of every variable on the classification, and is considered to have above-average influence when higher than 1.0. In our work, the variables with a VIP value of $>4.0$ were highlighted and selected. Their accurate $\mathrm{m} / \mathrm{z}$ information was used to search the matched lipid from database established by Waters or from LIPID MAPS (http://www.lipidmaps.org). The fragment ions were further utilized for confirmation of the marker. Significance of difference between multiple groups were analyzed by one-way ANOVA by SigmaStat (v3.5), and $P<0.05$ were considered statistically significant.

\section{Additional file}

Additional file 1: Table S1. Blood coagulation analysis of prothrombin time (PT), activated partial thromboplastin time (APTT) and thrombin time (TT) from healthy children $(\mathrm{HC})$ and Kawasaki disease (KD). Data are presented as mean \pm SD. Table S2. Detailed quantitative data and the statistical analysis of 19 TG molecular species contributing to the difference between the three groups. HC: healthy children; BT: fever patients from bacterial infections; KD: Kawasaki disease. Table S3. Detailed quantitative data and the statistical analysis of PC and LPC molecular species contributing to the difference between the three groups. HC: healthy children; BT: fever patients from bacterial infections; KD: Kawasaki disease. (DOCX 33 kb)

\section{Abbreviations}

APTT: activated partial thromboplastin time; BT: children with bacterial infections; BTNY: butyrophilin Y; CRP: C-reaction protein; DIC: disseminated intravascular coagulation; ESI: electrospray ionization; ESR: erythrocyte sedimentation rate; FFA: free fatty acid; HC: healthy children; HPLC: high performance liquid chromatography; IL: interleukin; KD: Kawasaki disease; LPC: Iyso-phosphatidylcholine; MS: mass spectrometry; PC: phosphatidylcholine); PT: prothrombin time; Q-TOF: quadrupole-time of flight; SM: sphingomyelin; TEG: thromroelastography; TG: triglyceride; TNFa: tumor necrosis factor a; TT: thrombin time.

\section{Competing interests}

The authors declare that they have no competing interests.

\section{Authors' contributions}

CHX, XC and LZD designed and coordinated the study. ZWZ and LL performed the mass spectrometry analysis. HX, JTL and XJC performed the blood coagulation and blood biochemistry analysis. LJL helped with data collection. XC and ZWZ analyzed the raw data. CHX and XC drafted the manuscript. All authors read and approved the final manuscript.

\section{Acknowledgements}

This research is supported by grants from National Science and Technology Major Projects for Major New Drugs Innovation and Development (2013ZX09303003) and National Science Foundation of China (81202021). We thank Chao-Zhen Zhai for his technical assistance.

\section{Author details}

'Department of Cardiology, Children's Hospital, Zhejiang University School of Medicine, No.3333 Bin-Sheng Road, Bin-Jiang Dist, Hangzhou, Zhejiang 310052, China. ${ }^{2}$ Key Laboratory for Diagnosis and Treatment of Neonatal Diseases of Zhejiang Province, Hangzhou, Zhejiang, China. ${ }^{3}$ Beijing National Laboratory for Molecular Sciences, Key Laboratory of Analytical Chemistry for Living Biosystems, Institute of Chemistry, Chinese Academy of Sciences, Beijing, China.

Received: 21 October 2015 Accepted: 22 December 2015

Published online: 30 December 2015

\section{References}

1. Kawasaki T. Acute febrile mucocutaneous syndrome with lymphoid involvement with specific desquamation of the fingers and toes in children. Arerugi. 1967;16(3):178-222.

2. Wang W, Zhu WH, Qi YQ, Fu SL, Gong FQ. Cardiogenic shock: do not forget the possibility of Kawasaki disease. Turk J Pediatr. 2012;54(1):86-9.

3. Cho HJ, Yang SI, Kim KH, Kim JN, Kil HR. Cardiovascular risk factors of early atherosclerosis in school-aged children after Kawasaki disease. Korean J Pediatr. 2014:57(5):217-21. doi:10.3345/kjp.2014.57.5.217.

4. Gupta-Malhotra M, Gruber D, Abraham SS, Roman MJ, Zabriskie JB, Hudgins LC, et al. Atherosclerosis in survivors of Kawasaki disease. J Pediatr. 2009;155(4):572-7. doi:10.1016/j.jpeds.2009.04.054.

5. McCrindle BW, Mclntyre S, Kim C, Lin T, Adeli K. Are patients after Kawasaki disease at increased risk for accelerated atherosclerosis? J Pediatr. 2007;151(3):244-8. 8 e1.

6. Cheung YF, Yung TC, Tam SC, Ho MH, Chau AK. Novel and traditional cardiovascular risk factors in children after Kawasaki disease: implications for premature atherosclerosis. J Am Coll Cardiol. 2004;43(1):120-4.

7. Quehenberger O, Dennis EA. The human plasma lipidome. N Engl J Med. 2011;365(19):1812-23. doi:10.1056/NEJMra1 104901

8. Varbo A, Benn M, Tybjaerg-Hansen A, Jorgensen AB, Frikke-Schmidt R, Nordestgaard BG. Remnant cholesterol as a causal risk factor for ischemic heart disease. J Am Coll Cardiol. 2013;61(4):427-36. doi:10.1016/j.jacc.2012.08.1026. 
9. Hopkins PN, Heiss G, Ellison RC, Province MA, Pankow JS, Eckfeldt JH, et al. Coronary artery disease risk in familial combined hyperlipidemia and familial hypertriglyceridemia: a case-control comparison from the National Heart, Lung, and Blood Institute Family Heart Study. Circulation. 2003;108(5):519-23.

10. Goldberg IJ, Eckel RH, McPherson R. Triglycerides and heart disease: still a hypothesis? Arterioscler Thromb Vasc Biol. 2011;31(8):1716-25. doi:10.1161/ATVBAHA.111.226100.

11. Jorgensen AB, Frikke-Schmidt R, West AS, Grande P, Nordestgaard BG, Tybjaerg-Hansen A. Genetically elevated non-fasting triglycerides and calculated remnant cholesterol as causal risk factors for myocardial infarction. Eur Heart J. 2013;34(24):1826-33. doi:10.1093/eurheartj/ehs431.

12. Nordestgaard BG, Varbo A. Triglycerides and cardiovascular disease. Lancet. 2014;384(9943):626-35. doi:10.1016/S0140-6736(14)61177-6.

13. Ayusawa M, Sonobe T, Uemura S, Ogawa S, Nakamura Y, Kiyosawa N, et al. Revision of diagnostic guidelines for Kawasaki disease (the 5 th revised edition). Pediatr Int. 2005:47(2):232-4

14. Spiess BD, Tuman KJ, McCarthy RJ, DeLaria GA, Schillo R, Ivankovich AD Thromboelastography as an indicator of post-cardiopulmonary bypass coagulopathies. J Clin Monit. 1987;3(1):25-30.

15. Romlin BS, Wahlander H, Berggren H, Synnergren M, Baghaei F, Nilsson K, et al. Intraoperative thromboelastometry is associated with reduced transfusion prevalence in pediatric cardiac surgery. Anesth Analg. 2011;112(1):30-6. doi:10.1213/ANE.0b013e3181fe4674.

16. Ishikawa M, Maekawa K, Saito K, Senoo Y, Urata M, Murayama M, et al. Plasma and serum lipidomics of healthy white adults shows characteristic profiles by subjects' gender and age. PLoS One. 2014;9(3):e91806. doi:10.1371/journal.pone.0091806.

17. Hara T, Mizuno Y, Akeda H, Fukushige J, Ueda K, Aoki T, et al. Thrombocytopenia: a complication of Kawasaki disease. Eur J Pediatr. 1988;147(1):51-3.

18. Thapa R, Pramanik S, Dhar S, Kundu R. Neonatal Kawasaki disease with multiple coronary aneurysms and thrombocytopenia. Pediatr Dermatol. 2007:24(6):662-3.

19. Niwa K, Aotsuka H, Hamada H, Uchishiba M, Terai M, Niimi H. Thrombocytopenia: a risk factor for acute myocardial infarction during the acute phase of Kawasaki disease. Coron Artery Dis. 1995;6(11):857-64.

20. Nofech-Mozes Y, Garty BZ. Thrombocytopenia in Kawasaki disease: a risk factor for the development of coronary artery aneurysms. Pediatr Hematol Oncol. 2003;20(8):597-601.

21. Parvathy VK, Manuel AD, Criton S, Rajesh G. Kawasaki disease with DIC as a complication. Indian J Pediatr. 2007;74(11):1049.

22. Lee $\mathrm{SH}$, Jeon $\mathrm{YH}$, Yang HJ, Pyun BY. Pleural effusion and disseminated intravascular coagulopathy: the rarely reported complications of kawasaki disease. Clin Pediatr (Phila). 2010;49(6):598-600. doi:10.1177/0009922809356468.

23. Saraswathi $\mathrm{V}$, Hasty $\mathrm{AH}$. The role of lipolysis in mediating the proinflammatory effects of very low density lipoproteins in mouse peritoneal macrophages. J Lipid Res. 2006;47(7):1406-15.

24. Wang L, Gill R, Pedersen TL, Higgins LJ, Newman JW, Rutledge JC. Triglyceride-rich lipoprotein lipolysis releases neutral and oxidized FFAs that induce endothelial cell inflammation. J Lipid Res. 2009;50(2):204-13. doi:10.1194/jlr.M700505-JLR200.

25. Teslovich TM, Musunuru K, Smith AV, Edmondson AC, Stylianou IM, Koseki $\mathrm{M}$, et al. Biological, clinical and population relevance of 95 loci for blood lipids. Nature. 2010;466(7307):707-13. doi:10.1038/nature09270.

26. Do R, Willer CJ, Schmidt EM, Sengupta S, Gao C, Peloso GM, et al. Common variants associated with plasma triglycerides and risk for coronary artery disease. Nat Genet. 2013;45(11):1345-52. doi:10.1038/ng.2795.

27. Mostafavi SN, Barzegar E, Manssori NS, Kelishadi R. First report on the lipid profile late after kawasaki disease in Iranian children. Int J Prev Med. 2014;5(7):820-4.

28. Duan C, Du ZD, Wang Y, Jia LQ. Effect of pravastatin on endothelial dysfunction in children with medium to giant coronary aneurysms due to Kawasaki disease. World J Pediatr. 2014;10(3):232-7. doi:10.1007/s12519-014-0498-5.

29. El-Hazmi MA, Warsy AS. Evaluation of serum cholesterol and triglyceride levels in 1-6-year-old Saudi children. J Trop Pediatr. 2001;47(3):181-5.

30. Christensen B, Glueck C, Kwiterovich P, Degroot I, Chase G, Heiss G, et al. Plasma cholesterol and triglyceride distributions in 13,665 children and adolescents: the Prevalence Study of the Lipid Research Clinics Program. Pediatr Res. 1980;14(3):194-202.

31. Marwaha RK, Khadgawat R, Tandon N, Kanwar R, Narang A, Sastry A, et al. Reference intervals of serum lipid profile in healthy Indian school children and adolescents. Clin Biochem. 2011;44(10-11):760-6. doi:10.1016/j.clinbiochem.2011.05.011.
32. Bird SS, Marur VR, Sniatynski MJ, Greenberg HK, Kristal BS. Serum lipidomics profiling using LC-MS and high-energy collisional dissociation fragmentation: focus on triglyceride detection and characterization. Anal Chem. 2011:83(17):6648-57. doi:10.1021/ac201195d.

33. Quehenberger $\mathrm{O}$, Armando AM, Brown AH, Milne SB, Myers DS, Merrill AH, et al. Lipidomics reveals a remarkable diversity of lipids in human plasma. J Lipid Res. 2010;51(11):3299-305. doi:10.1194/jlr.M009449.

34. Stegemann C, Pechlaner R, Willeit $P$, Langley SR, Mangino M, Mayr U, et al. Lipidomics profiling and risk of cardiovascular disease in the prospective population-based Bruneck study. Circulation. 2014;129(18):1821-31. doi:10.1161/CIRCULATIONAHA.113.002500.

35. Bellis C, Kulkarni H, Mamtani M, Kent JW, Jr., Wong G, Weir JM et al. Human Plasma Lipidome Is Pleiotropically Associated with Cardiovascular Risk Factors and Death. Circ Cardiovasc Genet. 2014. doi:10.1161/CIRCGENETICS.1k14.000600.

36. Kulkarni H, Meikle PJ, Mamtani M, Weir JM, Almeida M, Diego V, et al. Plasma lipidome is independently associated with variability in metabolic syndrome in Mexican American families. J Lipid Res. 2014;55(5):939-46. doi:10.1194/jlr.M044065.

37. Weir JM, Wong G, Barlow CK, Greeve MA, Kowalczyk A, Almasy L, et al. Plasma lipid profiling in a large population-based cohort. J Lipid Res. 2013;54(10):2898-908. doi:10.1194/jlr.P035808.

38. Pietilainen $\mathrm{KH}$, Sysi-Aho M, Rissanen A, Seppanen-Laakso T, Yki-Jarvinen $\mathrm{H}$, Kaprio J, et al. Acquired obesity is associated with changes in the serum lipidomic profile independent of genetic effects-a monozygotic twin study. PLoS One. 2007;2(2):e218.

39. Kotronen A, Velagapudi VR, Yetukuri L, Westerbacka J, Bergholm R, Ekroos K, et al. Serum saturated fatty acids containing triacylglycerols are better markers of insulin resistance than total serum triacylglycerol concentrations. Diabetologia. 2009;52(4):684-90. doi:10.1007/s00125-009-1282-2.

40. Wang Y, Wang W, Gong F, Fu S, Zhang Q, Hu J, et al. Evaluation of intravenous immunoglobulin resistance and coronary artery lesions in relation to Th1/Th2 cytokine profiles in patients with Kawasaki disease. Arthritis Rheum. 2013;65(3):805-14. doi:10.1002/art.37815.

41. Lee Y, Schulte DJ, Shimada K, Chen S, Crother TR, Chiba N, et al. Interleukin1 beta is crucial for the induction of coronary artery inflammation in a mouse model of Kawasaki disease. Circulation. 2012;125(12):1542-50. doi:10.1161/CIRCULATIONAHA.111.072769.

42. Grunfeld C, Dinarello CA, Feingold KR. Tumor necrosis factor-alpha, interleukin-1, and interferon alpha stimulate triglyceride synthesis in HepG2 cells. Metabolism. 1991;40(9):894-8.

43. Nonogaki K, Fuller GM, Fuentes NL, Moser AH, Staprans I, Grunfeld C, et al. Interleukin-6 stimulates hepatic triglyceride secretion in rats. Endocrinology. 1995;136(5):2143-9.

44. Delgado-Lista J, Garcia-Rios A, Perez-Martinez P, Solivera J, Yubero-Serrano EM, Fuentes F, et al. Interleukin 1B variant -1473G/C (rs1143623) influences triglyceride and interleukin 6 metabolism. J Clin Endocrinol Metab. 2011;96(5):E816-20. doi:10.1210/jc.2010-2455.

45. Piche ME, Lemieux S, Weisnagel SJ, Corneau L, Nadeau A, Bergeron J. Relation of high-sensitivity C-reactive protein, interleukin-6, tumor necrosis factor-alpha, and fibrinogen to abdominal adipose tissue, blood pressure, and cholesterol and triglyceride levels in healthy postmenopausal women. Am J Cardiol. 2005;96(1):92-7.

46. Gottsater A, Forsblad J, Matzsch T, Persson K, Ljungcrantz I, Ohlsson K, et al. Interleukin-1 receptor antagonist is detectable in human carotid artery plaques and is related to triglyceride levels and Chlamydia pneumoniae IgA antibodies. J Intern Med. 2002;251(1):61-8.

47. Fukunaga H, Kishiro M, Akimoto K, Ohtsuka Y, Nagata S, Shimizu T. Imbalance of peroxisome proliferator-activated receptor gamma and adiponectin predisposes Kawasaki disease patients to developing atherosclerosis. Pediatr Int. 2010;52(5):795-800. doi:10.1111/j.1442-200X.2010.03160.x.

48. Sarafian MH, Gaudin M, Lewis MR, Martin FP, Holmes E, Nicholson JK, et al. Objective set of criteria for optimization of sample preparation procedures for ultra-high throughput untargeted blood plasma lipid profiling by ultra performance liquid chromatography-mass spectrometry. Anal Chem. 2014:86(12):5766-74. doi:10.1021/ac500317c. 